\title{
GLOBAL OPTIMISATION OF CAR FRONT-END GEOMETRY TO MINIMISE PEDESTRIAN HEAD INJURY LEVELS
}

\author{
Kianifar, Mohammed Reza; Campean, Felician \\ University of Bradford, Automotive Research Centre
}

\begin{abstract}
The paper presents a multidisciplinary design optimisation strategy for car front-end profile to minimise head injury criteria across pedestrian groups. A hybrid modelling strategy was used to simulate the carpedestrian impact events, combining parametric modelling of front-car geometry with pedestrian models for the kinematics of crash impact. A space filling response surface modelling strategy was deployed to study the head injury response, with Optimal Latin Hypercube (OLH) Design of Experiments sampling and Kriging technique to fit response models. The study argues that the optimisation of the front-end car geometry for each of the individual pedestrian models, using evolutionary optimisation algorithms is not an effective global optimization strategy as the solutions are not acceptable for other pedestrian groups. Collaborative Optimisation (CO) multidisciplinary design optimisation architecture is introduced instead as a global optimisation strategy, and proven that it can enable simultaneous minimisation of head injury levels for all the pedestrian groups, delivering a global optimum solution which meets the safety requirements across the pedestrian groups.
\end{abstract}

Keywords: Computational design methods, Optimisation, Multidisciplinary Design Optimisation, Computer Aided Design (CAD), Passive Pedestrian Safety

\section{Contact:}

Campean, Felician

University of Bradford

School of Engineering

United Kingdom

F.Campean@bradford.ac.uk 


\section{INTRODUCTION}

Despite the continuous decline in the number of road fatal accidents over the last two decades, a large number of road fatalities are still reported worldwide every year. For instance, in Great Britain in the year ending in June 2018, 1,770 road deaths have been recorded, which is a level similar to that seen since 2012, and a mere 35\% reduction compared to 2008 (DfT, 2018). Investigations into the severity of the injuries related to the car-pedestrian occurrences shown that the major cause of fatality in such incidents is mainly linked to the head injuries, while the lower body injuries are generally attached to the long-term consequences (Kausalyah, et al., 2014; Zhao et al., 2010; Carter et al., 2005). Various circumstances might affect the severity of a pedestrian-car casualty, such as vehicle speed, pre-crash pedestrian stance and angle of collision (Untaroiu et al., 2010; Yao et al., 2008; Liu \& Yang, 2003). Liu and Yang (2003) discussed that the vehicle front geometry affects the severity of the pedestrian injuries for about $84 \%$ of the incidents. Thus, the design of front-end geometry of a car not only affects the vehicle aerodynamics, but also passively influences the pedestrian safety by determining the post-impact kinematics of the pedestrian (Kausalyah et al., 2014).

Most of the available papers in the area of passive pedestrian safety design have attempted to improve the modelling strategies for car-pedestrian events. Several studies have reported on modelling the pedestrian kinematics after the crash using multi-body dynamic environment, by either MADYMO (Kausalyah et al., 2014; Zhao et al., 2010; Carter et al., 2005) or LS DYNA (Ptak et al., 2010; Shen et al., 2008), both packages offering a fast simplified yet reasonably accurate modelling process. Other studies have focussed on the application of Finite Element (FE) modelling strategies to model the frontcar stiffness / deformation after a pedestrian-car accident (Sun et al., 2015; Christensen et al., 2012; Zhang et al., 2011). Also, a number of authors suggested application of hybrid modelling strategies to combine the multi-body modelling techniques with FE models (Yao et al., 2008; Oh et al., 2008).

Several research studies reported in literature attempted to enhance the passenger passive safety by improving the front-car design, with the aim of minimising the pedestrians' head related injuries. Carter et al. (2005) used a multi-body dynamics environment to model the car-pedestrian impact for different pedestrian case studies. Given the difficulties identified with mathematical modelling of the head injury response due to non-linear nature of the problem, Carter tried to achieve an optimum solution for each case study with a specific movement condition in order to reduce the aggressiveness of the front-car geometry. The value of the study is that it considered both different body kinematics post impact (associated with child, male, and female anthropometrics) and also the pedestrian movements (i.e. far leg forward, and near leg forward rotating forward or backward). Zhao et al. (2010) employed a response surface modelling strategy to model the head injury levels for different pedestrian groups (i.e. child, female and male) based on seven front-car geometrical parameters. They have utilised a single-objective optimisation strategy, underpinned by a gradient-based optimisation algorithm, to find the optimum value of the front-end car design parameters for each of the pedestrian groups. The study showed that optimum solution for each of the pedestrian groups does not reduce the head injury levels of the other pedestrian groups. Similarly, Kausalyah et al. (2014) employed a Gaussian based response modelling strategy to model the head injury. However, they attempted to optimise the head injury levels for child and male pedestrian groups and applied a single objective optimisation strategy to minimise the head injury level using a genetic optimisation algorithm. The chid and male head injury responses were combined by using a simple weighting strategy.

Almost all of the relevant studies available in literature have catered for a single type of pedestrian group to enhance the passive pedestrian safety design. However, it is seen that optimisation of the front-end car geometry for a specific pedestrian profile cannot simultaneously ensure an optimal (and in some cases even acceptable) solution for other pedestrian groups, (i.e. due to different post-impact kinematics of children and adults bodies) (Kausalyah et al., 2014; Martin et al., 2011; Carter et al., 2005). None of the studies proposed an optimisation strategy to obtain a global solution for optimal protection across the pedestrian profiles (i.e. child, female and male). Given the importance to OEMs of determining the frontend geometrical design in the early stages of design process, since this design subsequently determines not only the pedestrian safety but also the car aerodynamics, manufacturing process and under-bonnet packaging, the current optimisation strategy needs to be fundamentally improved.

Coherent with the systems engineering approach, a method to deal with optimisation of complex engineering problems is to decompose the engineered system hierarchically into a number of 
subsystems. This hierarchical segmentation process helps to formulate the whole system behaviour using a multilevel hierarchy of system-constituent elements. Collaborative Optimisation (CO) (Kroo \& Manning, 2000; Braun et al., 1996) is a Multidisciplinary Design Optimization (MDO) framework that has been developed to support optimal system design architectures associated with hierarchical partitioning into subsystems or sub-problems. This hierarchical optimization methodology has shown promising performance in translating system-level targets down to the subsystems, while ensuring simultaneous consistency and optimality at the system-level (Kang et al., 2012). Given the capability of $\mathrm{CO}$ architecture to optimise the disciplines in parallel, combined with the CO's simple process of data-sharing among the disciplines, makes this approach a strong candidate for optimisation of the multi-attribute optimisation problems with a small number of sharing design variables (Kroo \& Manning, 2000; Braun et al., 1996).

The aim of this paper is to address the front-end car geometry optimisation problem in a more holistic approach by using a $\mathrm{CO}$ framework, to achieve a design that offers optimal protection across the range of pedestrian anthropometric profiles. This approach enables simultaneous formulation of the passive pedestrian safety related to minimising the head injury levels for each pedestrian profile in an optimisation framework to achieve a global optimum solution for the front-end car geometry that meets all the required safety constraints. The performance of the proposed optimisation strategy is evaluated in relation to the case study presented by Zhao et al. (2010), which motivated this research. The paper is organised as follows: a research methodology section introduces the car-pedestrian crash case study, including the explanation of the modelling process for the front-end car geometry and pedestrian kinematics, and the implemented response modelling strategy to model the head injury levels. The optimisation strategy to minimise the pedestrians' head injury is described next; this includes optimising the design problem for each pedestrian group using evolutionary algorithms, and the CO implementation to improve the optimisation strategy. The results obtained from the application of the CO framework are presented, followed by a discussion of the results and the broader implication of this development for the early stages of front-car design process.

\section{RESEARCH METHODOLOGY: CASE STUDY OVERVIEW}

Zhao et al. (2010) modelled the car-pedestrian post-event head injury using a hybrid Matlab / MADYMO simulation environment. The head injury criterion (HIC), calculated as the resultant linear acceleration of the centre of the gravity of head, was employed as the main indicator of injury severity, in line with common practice in the literature (Kausalyah et al., 2014; Carter et al., 2005). The frontcar geometry was modelled by a multi-body system including 7 active ellipsoids, as shown in Figure 1. This multi-body system has been utilised to represent different shapes of front-vehicle through changing 7 design parameters, as illustrated in Figure 2. The range of these 7 geometrical parameters was set to cover the possible front-car architecture of 3 different car types (i.e. small, family, and luxury). Table 1 summarises the standardised front-end car geometry optimisation parameters (i.e. the parameters' engineering values are transformed to the [-1 1] domain).

The TNO (i.e. The Netherlands Organization for Applied Scientific Research) ellipsoid models (TNO Automotive-1, 2005) was used to simulate the pedestrian post-event HIC, given the proven capability of these models to accurately predict the impact points on the vehicle and head (Lange et al., 2006). TNO models have been frequently employed in different studies to represent the kinematic dynamic response of different body shapes (Kausalyah et al., 2014; Untaroiu et al., 2009, 2010; Yao et al., 2008; Shen et al., 2008; Carter et al., 2005). In this paper, three TNO models are used to investigate the pedestrian post-event head injury for different pedestrian groups (as illustrated in Figure 3):

- '6-year child' TNO model: to simulate the child-pedestrian kinematics.

- '5th \% female' TNO model: to simulate the female-pedestrian kinematics.

- '95th \% male' TNO model: to simulate the male-pedestrian kinematics.

The car-pedestrian collision was simulated in the gravity field, while the designed front-car of $1100 \mathrm{~kg}$ mass collided with each of the 3 pedestrian models at $40 \mathrm{~km} / \mathrm{h}$, assuming a gait stance at an arbitrary walking distance to the car (see Zhao et al. (2010) for details regarding the simulation set-up). Figure 4 illustrates the methodology adopted in this research to mathematically model the front-car collision problem for the optimisation purposes. A Design of Experiments (DoE) strategy was employed to plan an efficient data collection strategy. Since information regarding the behaviour of the HIC response over the design parameters' design space was not available, a space-filling Optimal Latin Hypercube (OLH) 
sampling strategy was employed, to achieve good intersite and projection distances (Forrester et al., 2008) for the simulation experiments plan. The minimum number of required DoE points to adequately characterise a response behaviour for a problem with 7 design parameters is 70 test points (i.e. 10 multiplied by the number of design parameters) (Loeppky et al., 2009), however due to high non-linear nature of the response (Carter et al., 2005; Le Glatin, 2003) a DoE of 100 test points was used for this case study. These 100 test points were run for each of the 3 collision simulations in Matlab / MADYMO environment, i.e. for each of the car-pedestrian models.

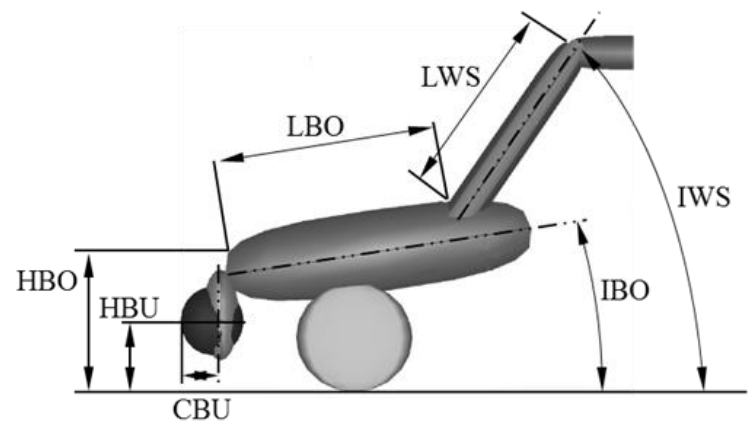

Figure 1. Parametric model of front-car geometry

Table 1. Summary of design parameters

$\begin{array}{lll}\text { Parameter } & \text { Symbol } & \text { Unit } \\ \text { Length of the bonnet } & \text { LBO } & \text { M } \\ \text { Height of the bonnet } & \text { HBO } & \text { M } \\ \text { Convexity of the bumper } & \text { CBU } & \text { M } \\ \text { Height of the bumper } & \text { HBU } & \text { M } \\ \text { Incline of the bonnet } & \text { IBO } & \mathrm{rad} \\ \text { Incline of the windscreen } & \text { IWS } & \mathrm{rad} \\ \text { Length of the windscreen } & \text { LWS } & \mathrm{M}\end{array}$

The data collected (HIC response from each simulation experiment) was used to derive metamodels for the head injury criterion response for each of the pedestrian groups (i.e. child, female and male). Given the non-linear behaviour of the HIC response, a Kriging model, which is a non-parametric modelling technique, was utilised. Kriging models have been frequently employed in literature to model complex response features, especially for computer-based problems (Gramacy \& Lian, 2012; Ben-Ari \& Steinberg, 2007). The main advantage of using Kriging method rather than other non-parametric modelling techniques such as Radial Basis Function (RBF) method, also frequently used for metamodels of head injury levels (see for example Sun et al., 2015; Kausalyah et al., 2014; Zhao et al., 2010), is that Kriging requires less hyperparameter tuning to deliver good model performance (Khan, 2011). The predictive performance of the Kriging models was evaluated by employing external diagnostic statistics using a set of validation test points (i.e. 10 additional measurements which were collected randomly across the parameters design space). The model validation criteria employed was based on residuals (i.e. the discrepancy between the predicted response values by the Kriging model $(\hat{y})$ and the actual response values from the MADYMO simulation (y)) over the validation set (v). Validation Root Mean Square Error (RMSE) for the validation test points (Harmann et al., 2013), calculated by Equation (1):

$$
\text { Validation RMSE }=\sqrt{\frac{1}{v} \cdot \sum_{i=1}^{v}\left(y_{i}-\hat{y}_{i}\right)^{2}}
$$

Relative Error is commonly used indicator for predication error over the validation set (Rango et al., 2013), calculated by Equation (2): 


$$
\text { Relative Error }(\%)=\sqrt{\frac{\sum_{i=1}^{v}\left(y_{i}-\hat{y}_{i}\right)^{2}}{\sum_{i=1}^{v} y_{i}^{2}}}
$$

The results in terms of HIC metamodel accuracy for each pedestrian group is summarised in Table 2 . In this table, Validation RMSE is in the same unit as HIC response, while the prediction error is calculated as the percentage of the average HIC value for each pedestrian profile. This table illustrates that all the delivered HIC response models are adequately accurate (i.e. around $5 \%$ prediction error).

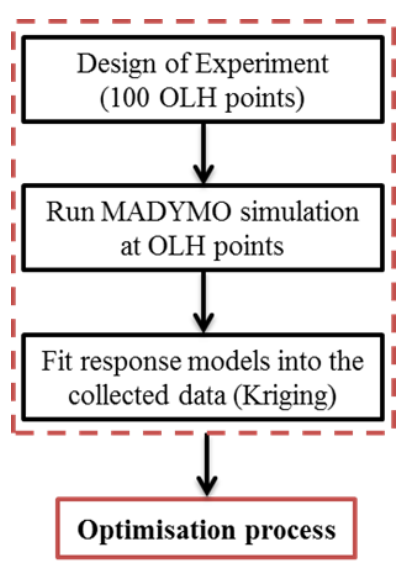

Figure 4. The modelling methodology

Table 2. Statistical properties of the HIC model for each pedestrian profile

$\begin{array}{llll} & \text { Child } & \text { Female } & \text { Male } \\ \text { Validation RMSE } & 91 & 151 & 63 \\ \text { ERROR }(\%) & 4.94 & 5.82 & 4.88\end{array}$

\section{COLLABORATIVE OPTIMISATION STRATEGY}

The optimisation strategy adopted in this research is to firstly find the optimum setting for the front-end car geometry design parameters in relation to each of the pedestrian groups using a Genetic Algorithm, which has been the common practice in this area (Mendoza-Vázquez et al., 2014; Carter et al., 2005). Then, describe the Collaborative Optimisation strategy and employ it to re-formulate the design problem as a global optimisation problem, to find an optimal setting for the front-end car design parameters for minimal predicted head injury across the pedestrian groups (i.e. child, female, and male).

\subsection{HIC minimisation for individual pedestrian models}

\subsubsection{Problem formulation}

A single-objective optimisation strategy was implemented to solve the front-car design optimisation problem for each of the pedestrian groups. The main objective of the optimisation problem was to minimise the HIC response for each of the pedestrian profiles, while meeting the design constraint of HIC $<1500$ for all the other pedestrian profiles, to avoid severe head injuries (Zhao et al., 2010). Thus, the objective function for each pedestrian group was formulated as in equation (3).

Objective:

Minimize $H I C(X)$

With respect to

$X=(L B O H B O C B U H B U I B O I W S L W S)$

Subject to:

$\operatorname{Convex}(X) \leq 0$

$L B \leq X \leq U B$

In Equation (6), $\mathrm{X}$ denotes the matrix of the design parameters (i.e. LBO, HBO, CBU, HBU, IBO, IWS, and LWS), as summarised in Table 1, and LB and UB are the lower and upper boundary limits for each 
design parameter. Additionally, a convex hull constraint was introduced as a design space constraint for the optimisation. Convex hull or convex envelope defines the minimal convex which contains all the tested DoE data points as the final design space. This constraint helps to avoid extrapolation in parts of design space unexplored through testing. Thus, Convex $X \leq 0$ ensures that the optimum front-car design solution is within the convex hull envelope.

Given that all the developed HIC response functions were explicitly available in Matlab programming environment, a global optimisation algorithm (i.e. based on the Genetic Algorithm Matlab function) was employed to solve the optimisation problem, given in Equation (3), in turn for each of the 3 considered pedestrian groups (i.e. child, female, and male pedestrian profiles).

\subsubsection{Results and discussion}

Table 3 summarises the optimisation solutions obtained by optimising the front-car optimisation problem. In this table, the parameters of the optimal design solution for each individual pedestrian group (as listed in the left column of the table) are given in coded values (i.e. [-1 1]), and the three columns on the right gives the predicted optimal HIC response.

Table 3. Optimum front- car design parameters for each pedestrian group

\begin{tabular}{|l|c|c|c|c|c|c|c|c|c|c|}
\cline { 2 - 11 } \multicolumn{1}{c|}{} & \multicolumn{9}{c|}{ Coded front-car design parameters } & \multicolumn{3}{c|}{ HIC } \\
\hline Optimum & LBO & HBO & CBU & HBU & IBO & IWS & LWS & Child & Female & Male \\
\hline Child & -0.25 & 0.56 & 0.26 & -0.19 & 0.86 & -0.14 & -0.11 & 837.21 & 1443.77 & 1162.38 \\
\hline Female & -0.04 & 0.03 & -0.60 & -0.06 & 0.84 & -0.67 & -0.18 & 1190.23 & 735.50 & 1298.17 \\
\hline Male & -0.36 & 0.46 & 0.24 & -0.54 & -0.63 & -0.25 & 0.62 & 1980.11 & 3037.46 & 714.69 \\
\hline
\end{tabular}

Table 3 indicates the complexity of the optimisation problem, since improving the design to protect one of the pedestrian groups leads to HIC performance degradation in relation to the other pedestrian groups, which was expected as similar findings were reported in literature (Kausalyah et al., 2014; Zhao et al., 2010).

For instance, optimising the design problem to minimise the HIC response for the male profile resulted into a very low HIC for a male pedestrian (i.e. HIC $=714.69$ ), whereas the design cannot meet the required safety for the other two pedestrian groups, i.e. the HIC responses calculated for these profiles are well above the threshold value. This severe design degradation in child and female HIC responses might be due to the huge difference between the male's optimum setting for height of the bumper (HBU), incline of the bonnet (IBO) and length of the windscreen (LWS) parameters. Similar trends are seen in relation to the optimal solutions for child and female pedestrian profiles. Both of these solutions improved the design safety for the considered pedestrian type, while severely affecting the safety of other groups. It should be emphasised that even though the optimum solution for child and female pedestrian profiles are overall feasible (i.e. meet the HIC constraint for the worst allowable design performance), it doesn't mean that either represent a global optimal solution.

\subsection{Simultaneous minimisation of HIC over all pedestrian models}

\subsubsection{Collaborative optimisation (CO) framework}

The CO optimisation framework is a bi-level multidisciplinary design optimisation strategy, principled on hierarchal decomposition of the optimisation problem into a system level and several sub-system levels (i.e. disciplines). The disciplines are optimised independently, while are also connected to each other through receiving copies of linking variables and sharing design variables at every iteration from the system level optimiser (i.e. copies of variables are also known as targets in many of references) (Braun et al., 1996). The duty of system-level optimiser is to minimise the design objective function, while the sub-system optimisers (i.e. at each discipline) are responsible to minimise the discrepancy between the system level targets and each discipline solutions in order to minimise the whole system inconsistency (Kroo \& Manning, 2000). The mathematical formulation of the system-level optimisation problem using the $\mathrm{CO}$ architecture is given in Equation (4).

In equation (4), $X$ is the matrix of design parameters, $X_{i}{ }^{L}$ is the matrix of target values transferred from the sub-system level i (i.e. discipline i), $\mathrm{N}$ is the number of disciplines, and $h$ and $g$ are the vectors of equality and inequality constraints, respectively. In the $\mathrm{CO}$ optimisation formulation, $C^{c}$ is an equality constraint known as consistency constraint which is responsible of preserving the whole 
system consistency by ensuring both system-level and subsystem-level optimum solutions are agreeing on a single value.

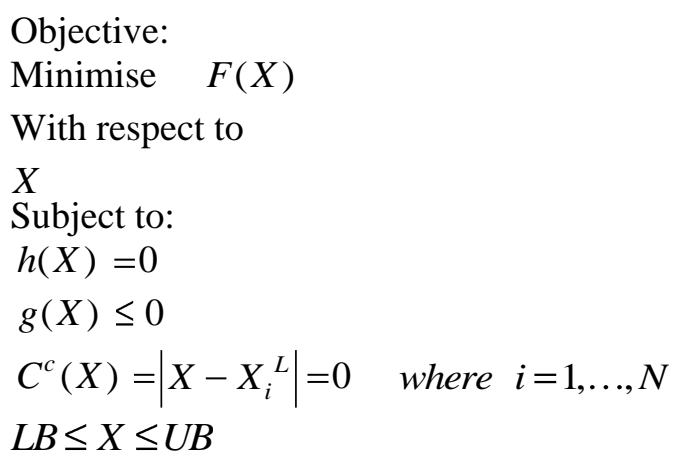

For each discipline, the objective is to meet the targets transferred from the system-level, i.e. by minimising the discrepancy between the subsystem-level and transferred system-level solutions, while the local constraints at each discipline are satisfied. Therefore, the optimisation problem at each discipline $i$ is formulated as in equation (5).

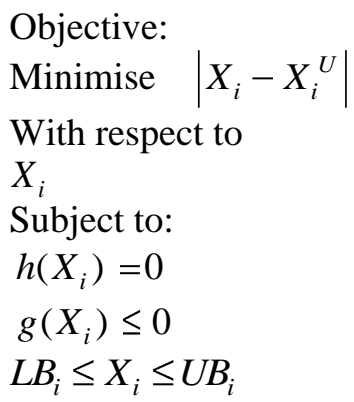

where $X_{i}$ is the matrix of design parameters at discipline $i, X_{i}^{U}$ is the matrix of target values for the design parameters at discipline i transferred from the system level optimisation, and $h$ and $g$ are the vectors of equality and inequality constraints at discipline $i$, respectively. Subsequently, the subsystem-level solutions will be returned to the system-level as the new targets for another iteration of the optimisation process.

\subsubsection{CO problem formulation}

The front-end car geometry optimisation problem attempts to minimise the HIC response for all the pedestrian groups, subject to the maximum HIC (i.e. HIC < 1500), in order to avoid severe head injuries. Therefore, the CO optimisation architecture can be associated with the front-car design optimisation problem by treating the optimisation problem for each pedestrian group as a subsystem or discipline, and the global optimisation problem over all the pedestrian groups as the system optimisation problem. Accordingly, the front-car optimisation problem can be formulated as a bi-level CO optimisation framework, consisting of a system level and three subsystem levels.

At the system level optimization, the main objective is to minimise the HIC response over all 3 pedestrian groups, whereas the overall system consistency is ensured by the consistency constraint $\left(C^{c}\right)$, as illustrated in Equation (6).

Objective:

Minimise $\quad \sum_{i=1}^{3} w_{i} \times H I C_{i}(X)$

With respect to

$X=($ LBO HBOCBU HBU IBOIWS LWS $)$

Subject to:

$$
\begin{aligned}
& C^{c}(X)=\left|X-X_{i}{ }^{L}\right|=0 \quad \text { where } i=1, \ldots, 3 \\
& L B \leq X \leq U B
\end{aligned}
$$

In Equation (6), $X$ indicates the matrix of front-car design parameters, $X_{i}{ }^{L}$ denotes the cascaded optimisation solution from subsystem $i, w_{i}$ defines the weight considered for each pedestrian group $i$, $H I C_{i}$ is the Kriging response model fitted for the pedestrian group i, $C^{c}$ is the consistency constraint, 
and $L B$ and $U B$ are defining the boundary limits for the design parameters. In the system level, the objective function is formulated as the weighted sum of HIC responses for the 3 pedestrian profiles, where a weight is introduced for each HIC response, reflecting the overall optimisation preference to enhance each pedestrian design safety. However, since in this study it is important to minimise the $\mathrm{HIC}$ response for all the pedestrian profiles, all the responses are equally weighted $\left(w_{i}=1\right)$.

At the subsystems level, the main objective for each subsystem i (i.e. for each pedestrian profile i) was to ensure consistency with the cascaded system level targets, by minimizing the discrepancy between the subsystem solutions $X$ and the cascaded down targets from the system level $X_{i}^{U}$, while satisfying the nonlinear constraints for HIC response and convex hull for the pedestrian profile $i$, within the design space boundary limits, as illustrated in Equation (7).

Objective:

Minimise $\left|X-X_{i}^{U}\right|$

With respect to

$X=($ LBO HBOCBU HBU IBOIWS LWS $)$

Subject to:

$$
\begin{aligned}
& \operatorname{HIC}_{i}(X) \leq 1500 \\
& \text { Convex }_{i}(X) \leq 0 \\
& L B \leq X \leq U B
\end{aligned}
$$

Overall, this design optimisation problem has 7 design parameters which link the system level to each of the subsystems, while there is also a coupling between subsystems as they share the 7 design parameters, i.e. by the consistency constraint applied at the system level. Given that all the HIC response functions are explicitly available, and the engineering preference for computation speed / fast convergence, a gradient based sequential quadratic programming (SQP) algorithm, based on the fmincon Matlab function, was employed to solve the CO problem at both system and subsystem levels. Figure 5 illustrates the organisation of the implemented $\mathrm{CO}$ framework for the front-end car design optimisation problem.

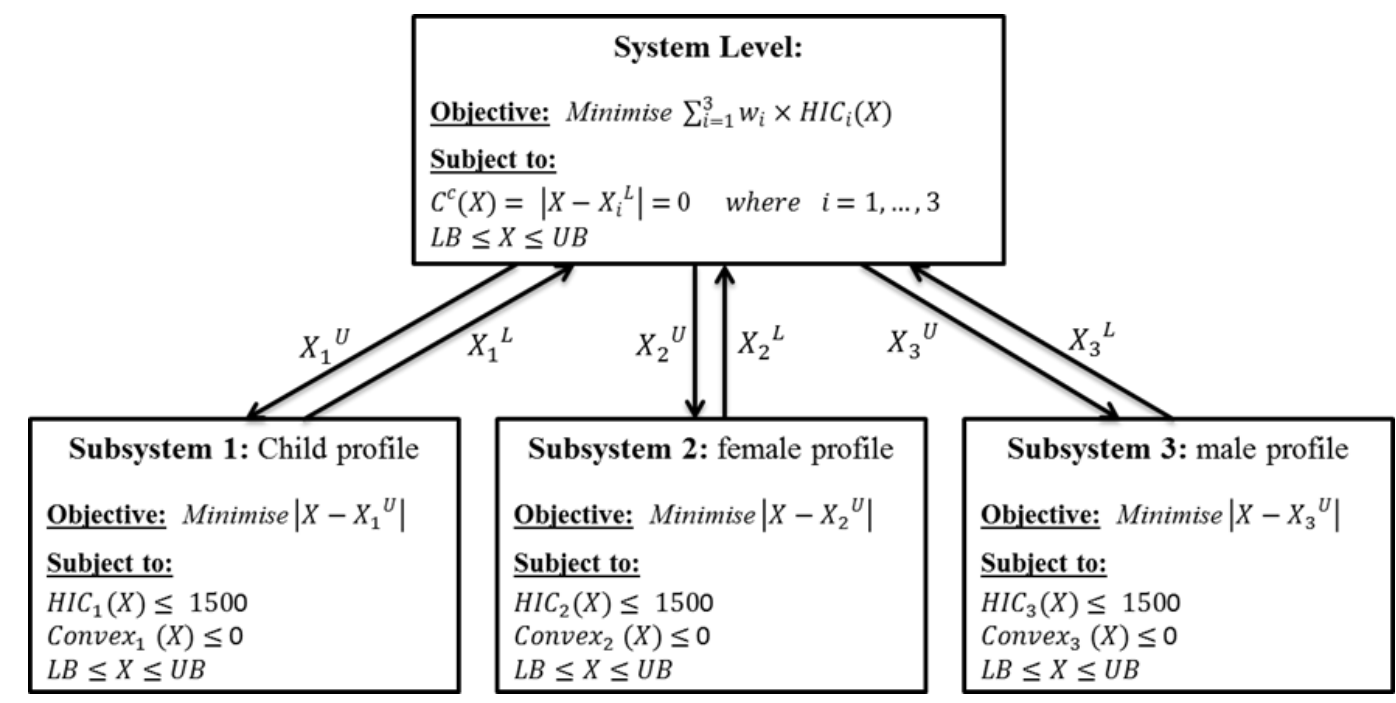

Figure 5: Decomposition and information flow of the CO process for the front-car design optimisation problem

\subsubsection{Results and discussion}

Table 4 summarises the global optimum solution for the front-car design parameters in coded values, optimised using the $\mathrm{CO}$ framework to simultaneously reduce the head injury risk among all the three considered pedestrian groups. With reference to the optimal HIC responses illustrated in Table 4, comparing these with the results in Table 3, it is seen that:

1. While the $\mathrm{CO}$ results are slightly worse than the best individual results seen in Table 3 , the average HIC (calculated across the pedestrian groups) is better than any of the results in Table 3 (specifically $6.3 \%$ better than the female, $13.5 \%$ better than the Child, and $88 \%$ better than the male optimal HIC results. 
2. The safety margin (calculated as distance to the maximum allowable HIC threshold of 1500) has increase with $3.7 \%$ compared to the child and $13.5 \%$ compared to the female single optimisation solution.

Table 4. Optimum front- car design parameters for all three pedestrian groups

\begin{tabular}{|l|c|c|c|c|c|c|c|c|c|c|}
\cline { 2 - 11 } \multicolumn{1}{c|}{} & \multicolumn{9}{c|}{ Coded front-car design parameters } & \multicolumn{3}{c|}{ HIC } \\
\hline Optimum & LBO & HBO & CBU & HBU & IBO & IWS & LWS & Child & Female & Male \\
\hline CO Solution & -0.09 & 0.12 & -0.41 & -0.12 & 0.84 & -0.63 & -0.11 & 1060.33 & 792.77 & 1181.29 \\
\hline
\end{tabular}

\section{SUMMARY, CONCLUSIONS AND FUTURE WORK}

This paper has demonstrated that the collaborative optimisation strategy can deliver strong benefits in formulation and optimisation of complex pedestrian-friendly front-car geometry design problem.

The Kriging response modelling strategy (not commonly employed for car-pedestrian impact metamodelling studies) was shown to be efficient for modelling the nonlinear pedestrian- vehicle impacts. All the HIC fitted responses modelled are accurate in relation to trends and prediction error (less than 6\%), thus suitable for optimisation purposes.

The results obtained from optimisation of HIC response for individual pedestrian groups, summarised in Table 3, demonstrate that an optimum design solution for one of the pedestrian groups will not necessary result in a safe or optimal solution for all pedestrian groups. Therefore, a new multidisciplinary design optimisation strategy was introduced to enable simultaneous optimisation of frontcar design problem across different pedestrian groups. In the proposed strategy, the complex optimisation problem was hierarchically decomposed and solved using a bi-level collaborative optimisation framework, where the safety objectives over all three pedestrian groups were formulated in the system-level and the safety objectives for each of the pedestrian groups are formulated in the sub-system level. The achieved global solution, illustrated in Table 4, is overall significantly better than the single objective optimisation solution, proving the effectiveness of the CO strategy.

The collaborative optimisation framework can be further extended to integrate the passive pedestrian safety problem along with other engineering objectives, e.g. aerodynamics and weight, which are also affected by the same front-car design parameters. The collaborative optimisation strategy can also be employed to evaluate and optimise more complex system designs involving combinations of pedestrian safety technologies (e.g. including active bonnet systems featuring pedestrian airbags) to offer enhanced protection across multiple cases of impact circumstances.

\section{REFERENCES}

Ben-Ari, E. N. and Steinberg, D. M. (2007), "Modeling Data from Computer Experiments: An Empirical Comparison of Kriging with MARS and Projection Pursuit Regression," Qual. Eng., Vol. 19 No. 4, pp. 327-338. http://doi.dx.org/10.1080/08982110701580930.

Braun, R. D., Moore, A. A. and Kroo, I. M. (1996a), "Use of the Collaborative Optimization Architecture for Launch Vehicle Design”, AIAA NASA and ISSMO Symposium on Multidisciplinary Analysis and Optimisation, AIAA Vol. 96, 4018, pp. 316-318, http://doi.dx.org/10.2514/6.1996-4018.

Braun, R., Gage, P., Kroo, I. and Sobiesk, I. (1996b), "Implementation and Performance issues in Collaborative Optimization,” Proc. 6th AIAA/USAF/NASA/ISSMO Multidiscip. Anal. Optim. Symp., Vol. AIAA 1996. http://doi.dx.org/10.1.1.45.6393.

Carter, E., Ebdon, S. and Neal-Sturgess, C. (2005), "Optimization of passenger car design for the mitigation of pedestrian head injury using a genetic algorithm," in Proceedings of the 2005 conference on Genetic and evolutionary computation - GECCO '05, pp. 2113-2120. http://doi.dx.org/10.1145/1068009.1068358.

Christensen, J., Bastien, C. and Blundell, M. V. (2012), "Effects of roof crush loading scenario upon body in white using topology optimisation,” Int. J. Crashworthiness, Vol. 17 No. 1, pp. 29-38. http://doi.dx.org/10.1080/13588265.2011.625640.

De Lange, L., Rooij, V., Happee, R. and Liu, X. J. (2006), "Validation of Human Pedestrian Models Using Laboratory Data as well as Accident Reconstruction," in Expert Symposium on Accident Research (ESAR).

Department for Transport (2018), "Reported road casualties in Great Britain: quarterly provisional estimates year ending June 2018", available from https://www.gov.uk/transport/road-accidents-and-serious-accidents, accessed 24/11/2018.

Forrester, A., Sobester, D. A. and Keane, A. (2008), "Engineering Design via Surrogate Modelling: A Practical Guide". John Wiley and Sons. 
Gramacy, R. B. and Lian, H. (2012), “Gaussian Process Single-Index Models as Emulators for Computer Experiments,” Technometrics, Vol. 54 No. 1, pp. 30-41. http://doi.dx.org/10.1080/00401706.2012.650527.

Hartmann, B., Baumann, W. and Nelles, O. (2013), “Axes-Oblique Partitioning of Local Model Networks for Engine Calibration," in Design of Experiments (DoE) in Engine Development, pp. 92-106.

Joseph, V. R., Hung, Y. and Sudjianto, A. (2008), "Blind Kriging: A New Method for Developing Metamodels," J. Mech. Des., Vol. 130 No. 3. http://doi.dx.org/10.1115/1.2829873.

Kang, N., Kokkolaras, M. and Papalambros, P. (2012), “Optimal Design of Commercial Vehicle Systems Using Analytical Target Cascading," 12th AIAA Aviat. Technol. Integr. Oper. Conf. 14th AIAA/ISSMO Multidiscip. Anal. Optim. Conf. http://doi.dx.org/10.2514/6.2012-5524.

Kausalyah, V., Shasthri, S., Abdullah, K. A., Idres, M. M., Shah, Q. H. and Wong, S. V. (2014a), "Development of Economical Vehicle Model for Pedestrian Friendly Front End Profile Study," Int. J. Simul. Model, Vol. 13 No. 4, pp. 419-432.

Kausalyah, V., Shasthri, S., Abdullah, K. A., Idres, M. M., Shah, Q. H. and Wong, S. V. (2014b), “Optimisation of vehicle front-end geometry for adult and pediatric pedestrian protection," Int. J. Crashworthiness, Vol. 19 No. 2, pp. 153-160. http://doi.dx.org/10.1080/13588265.2013.879506.

Khan, M. A. Z. (2011), "Transient engine model for calibration using two-stage regression approach," $\mathrm{PhD}$ Thesis, Loughborough University.

Kroo, I. and Manning, V. (2000), "Collaborative Optimisation: Status and directions," in 8th AIAA/NASA/ISSMO Symposium on Multidisciplinary Analysis and Optimization.

Le Glatin, N. G. (2003), "Design of experiments analysis study of real world pedestrian-vehicle accident simulation scenarios", School of Engineering, Coventry University.

Liu, X. and Yang, J. (2013), "Effects of vehicle impact velocity and front-end structure on dynamic responses of child pedestrians", Traffic Inj. Prev., Vol. 4 No. 4, pp. 337-344. http://doi.dx.org/10.1080/714040491.

Loeppky, J. L., Sacks, J. and Welch, W. J. (2009), "Choosing the Sample Size of a Computer Experiment: A Practical Guide”, Technometrics, Vol. 51 No. 4, pp. 366-376. http://doi.dx.org/10.1198/TECH.2009.08040.

Martin, J. L., Lardy, A. and Laumon, B. (2011), "Pedestrian injury patterns according to car and casualty characteristics in france”, Ann. Adv. Automot. Med., Vol. 55, pp. 137-146.

Mendoza-Vázquez, M., Jakobsson, L., Davidsson, J., Brolin, K. and Östmann, M. (2014), "Evaluation of Thoracic Injury Criteria for THUMS Finite Element Human Body Model Using Real-World Crash Data", in IRCOBI Conference 2014, pp. 528-541.

Oh, C., Kang, Y. and Kim, W. (2008), "Assessing the safety benefits of an advanced vehicular technology for protecting pedestrians”, Accid. Anal. Prev., Vol. 40 No. 3, pp. 935-42. http://doi.dx.org/10.1016/j.aap.2007.10.010.

Ptak, M., Karliński, J. and Kopczyński, A. (2010), “Analysis of pedestrian passive safety with the use of numerical simulation”, J. Kones, Vol. 17 No. 1, pp. 337-342.

Rango, J., Schnorbus, T., Kwee, H., Beck, R., Kinoo, B., Arthozoul, S. and Zhang, M. (2013), “Comparison of Different Approaches for Global Modeling of Combustion Engines," in Design of Experiments (DoE) in Engine Development, pp. 70-91.

Shen, J., Jin, X. L. and Zhang, X. Y. (2008), "Simulated evaluation of pedestrian safety for flat-front vehicles", Int. J. Crashworthiness, Vol. 13 No. 3, pp. 247-254. http://doi.dx.org/10.1080/13588260801933584.

Sun, G., Lv, X., Fang, J., Gu, X. and Li, Q. (2015), "Reliability-based design optimization of vehicle front-end structure for pedestrian lower extremity protection," in 11th World Congress on Structural and Multidisciplinary Optimisation.

TNO Automotive-1 (2005), MADYMO Human Models Manual, V6.2.2, TNO Automotive, The Netherlands.

Untaroiu, C. D., Crandall, J. R., Takahashi, Y., Okamoto, M., Ito, O. and Fredriksson, R. (2010), "Analysis of running child pedestrians impacted by a vehicle using rigid-body models and optimization techniques", Saf. Sci., Vol. 48 No. 2, pp. 259-267. http://doi.dx.org/10.1016/j.ssci.2009.09.003.

Untaroiu, C. D., Meissner, M. U., Crandall, J. R., Takahashi, Y., Okamoto, M. and Ito, O. (2009), "Crash reconstruction of pedestrian accidents using optimization techniques”, Int. J. Impact Eng., Vol. 36 No. 2 , pp. 210-219. http://doi.dx.org/10.1016/j.ijimpeng.2008.01.012

Yao, J., Yang, J. and Otte, D. (2008), "Investigation of head injuries by reconstructions of real-world vehicleversus-adult-pedestrian accidents", Saf. Sci., Vol. 46 No. 7, pp. 1103-1114. http://doi.dx.org/10.1016/j.ssci.2007.06.021.

Zhang, J., Chen, G. and Tang, H. (2011), "Parametric design and structural improvements to optimise frontal crashworthiness of a truck", Int. J. Crashworthiness, Vol. 16 No. 5, pp. 501-509. http://doi.dx.org/10.1080/13588265.2011.611395.

Zhao, Y, Rosala, G. F., Campean, I. F. and Day, A. J. (2010), “A response surface approach to front-car optimisation for minimising pedestrian head injury levels", Int. J. Crashworthiness, Vol. 15 No. 2 pp. 143150. http://doi.dx.org/10.1080/13588260903094392. 